\title{
Deep odontogenic infections-identifying risk factors for nosocomial pneumonia
}

\author{
Niina Rautaporras ${ }^{1}$ (D) Jussi Furuholm ${ }^{1} \cdot$ Johanna Uittamo $^{1} \cdot$ Mikko Saloniemi $^{1} \cdot$ Tuukka Puolakka $^{2,3} \cdot$ Johanna Snäll $^{1}$
}

Received: 13 May 2020 / Accepted: 4 August 2020 / Published online: 13 August 2020

(C) The Author(s) 2020

\begin{abstract}
Objectives To evaluate occurrence and risk factors for pneumonia in patients with deep odontogenic infection (OI). Materials and methods All patients treated for deep OIs and requiring intensive care and mechanical ventilation were included. The outcome variable was diagnosis of nosocomial pneumonia. Primary predictor variables were re-intubation and duration of mechanical ventilation. The secondary predictor variable was length of hospital stay (LOHS). The explanatory variables were gender, age, current smoking, current heavy alcohol and/or drug use, diabetes, and chronic pulmonary disease.

Results Ninety-two patients were included in the analyses. Pneumonia was detected in 14 patients (15\%). It was diagnosed on postoperative day 2 to 6 (median 3 days, mean 3 days) after primary infection care. Duration of mechanical ventilation $(p=0.028)$ and LOHS $(p=0.002)$ correlated significantly with occurrence of pneumonia. In addition, re-intubation $(p=0.004)$ was found to be significantly associated with pneumonia; however, pneumonia was detected in $75 \%$ of these patients prior to re-intubation. Two patients $(2 \%)$ died during intensive care unit stay, and both had diagnosed nosocomial pneumonia. Smoking correlated significantly with pneumonia $(p=0.011)$.

Conclusion Secondary pneumonia due to deep OI is associated with prolonged hospital care and can predict the risk of death. Duration of mechanical ventilation should be reduced with prompt and adequate OI treatment, whenever possible. Smokers with deep OI have a significantly higher risk than non-smokers of developing pneumonia.

Clinical relevance Nosocomial pneumonia is a considerable problem in OI patients with lengthy mechanical ventilation. Prompt and comprehensive OI care is required to reduce these risk factors.
\end{abstract}

Keywords Odontogenic infection $\cdot$ Dental abscess $\cdot$ Deep neck infection $\cdot$ Nosocomial pneumonia $\cdot$ Risk factors

\section{Introduction}

Hospitalized patients with odontogenic infections (OIs) are at risk for life-threatening conditions and infection complications. These severe infections can lead to death despite accurate and multidisciplinary care [1-3]. Elderly patients [3, 4]

Niina Rautaporras

niina.rautaporras@helsinki.fi

1 Department of Oral and Maxillofacial Diseases, University of Helsinki and Helsinki University Hospital, P.O. Box 220, (Haartmaninkatu 4E), FI-00029 HUH Helsinki, Finland

2 Department of Emergency Medicine and Services, University of Helsinki and Helsinki University Hospital, Helsinki, Finland

3 Department of Anaesthesiology and Intensive Care Medicine, University of Helsinki and Helsinki University Hospital, Helsinki, Finland and patients with underlying disease [5, 6] are more susceptible to complicated infections. Generalized infection of dental origin can cause severe sepsis $[1,2]$ and spread to distant organs, causing disseminated infections such as endocarditis [5], mediastinitis [1, 2, 7-9], pleural emphysema [7], meningitis [10], subdural emphysema [10], brain abscess [11], necrotizing fasciitis $[9,12]$, and thrombophlebitis of the internal jugular vein (Lemierre's syndrome) [13]. Pneumonia is, however, a common secondary infection related to deep OI [2], and when it is acquired during hospitalization, the applicable term is nosocomial pneumonia.

Deep neck infection-associated pneumonia is reported to occur in $1-6 \%$ of hospitalized patients [2, 9, 14]. Patients' general condition affects prognosis, and patients with reduced immune defence have associated respiratory tract infection secondary to OI more often than in other populations [2]. Pneumonia complicates patient care in intensive care units (ICUs) in general; nearly two-thirds (64\%) of infections in this 
patient group are of respiratory origin [15], and as a nosocomial infection, the prevalence of ventilation-associated pneumonia (VAP) is 5-40\% [16]. Even though the definition of VAP and reporting standards vary [16, 17], pneumonia in patients with at least $48 \mathrm{~h}$ of mechanical ventilation and without preceding pneumonia is the most commonly used definition of VAP. Prolonged ICU stay [16, 18] and re-intubation episodes $[19,20]$ are known to predispose patients to respiratory infections. Especially extubation failure has been shown to be associated with pneumonia [20]. Thus, unplanned reintubation and emergency airway management predispose to these infections in general ICU patient populations.

Trismus, oedema, and pus formation in the oropharyngeal region as well as challenging airway management due to narrowed laryngeal space and limited visibility during intubation cause aspiration risk and predispose patients to respiratory infections. We aimed to clarify and evaluate the occurrence and special features of secondary pneumonia in patients treated for deep OI. We hypothesized that occurrence of secondary pneumonia is rather high in OI patients in general and that patient- and treatment-related factors would emerge.

\section{Materials and methods}

\section{Study design and inclusion criteria}

A retrospective cohort study was conducted in the Töölö Hospital Emergency Department of Helsinki University Hospital with a catchment area of 1.6 million inhabitants between 2015 and 2019. Patients who were treated in the oral and maxillofacial surgery's emergency service for deep OI (i.e., abscess and/or cellulitis of facial and/or neck region from dental origin) confirmed by oral and maxillofacial surgeons and required postoperative mechanical ventilation in the ICU were included in this study. Exclusions comprised patients with unclear infection, patients with other than odontogenic focus, patients with pneumonia at hospital admission, and patients treated without postoperative mechanical ventilation.

\section{Study variables}

The outcome variable was presence of pneumonia, defined as clinically suspected and radiologically confirmed pneumonia during OI treatment in ICU.

Primary predictor variables were re-intubation and duration of mechanical ventilation. The secondary predictor variable was length of hospital stay (LOHS).

Explanatory variables were gender, age, current smoking, current heavy alcohol and/or regular drug use, diabetes (DM), and chronic pulmonary disease. Consumption limits for heavy alcohol use were $\geq 12$ doses per week in women and $\geq 23$ doses in men, based on anamnestic information. In addition, bacterial stain in tracheal aspirates of pneumonia patients, airway management, need for late tracheostomy, and overall survival rate were recorded. Also, accurate information of pneumonia and administered antibiotic medications were gathered.

\section{Statistical analysis}

We used software package IBM SPSS for Macintosh (version 25.0, IBM Corp., Armonk, NY, USA) for statistical data analyses. For categorical variables, we evaluated differences in associations between the study groups with Pearson's chisquare test or with Fisher's exact test if expected values were below 5 . We used Student's $t$ test to analyse differences between groups for continuous variables. For multivariate analysis, we selected binomial logistic regression with occurrence of pneumonia as the dependent variable; of the predictors, age was categorized into tertiles, while the rest of the independent variables were dichotomous. Throughout the study, we considered $p$ values below 0.05 to be statistically significant.

\section{Results}

A total of 109 patients with oral and maxillofacial infection required treatment in ICU. Seventeen patients were excluded from the final analysis (Fig. 1). Thus, 92 patients remained to be analysed.

All 92 patients underwent surgical intervention of infection, i.e., incision and drainage of abscesses intra- and/or extra-orally and dental foci were identified and removed as a part of the surgical treatment if required. All patients were treated by oral and maxillofacial surgeons; thus, there was no delay in the focus identification and/or a focus tooth removal during hospital stay. One patient received tracheostomy at the primary stage of hospital care. The remaining 91 patients were intubated fiberoscopically or in a conventional manner. All options for airway management were available throughout the hospital stay. In addition to surgical treatment, all patients received antimicrobial medication (benzylpenicillin or cefuroxime combined with metronidazole or piperacillin combined with tazobactam).

Descriptive statistics of the 92 patients are presented in Table 1. Most of the patients were male (63 of 92, 68\%). Patients' age ranged from 18 to 88 years (mean 45 years, median 43 years). One-third of the patients were smokers (30 of $92,33 \%)$. Of the patients, 9 out of $92(10 \%)$ had DM, and $12(13 \%)$ had a history of chronic pulmonary disease, which was in all cases asthma and/or chronic obstructive pulmonary disease (COPD). Eleven patients (12\%) were heavy alcohol and/or drug abusers. LOHS ranged between 2 and 36 days (mean 7.3 days, median 6 days). Reintubation was required in 6 patients $(7 \%)$. Interestingly the number of immunocompromised patients was very low in our study; in 
Fig. 1 One hundred nine patients with maxillofacial infection treated in ICU

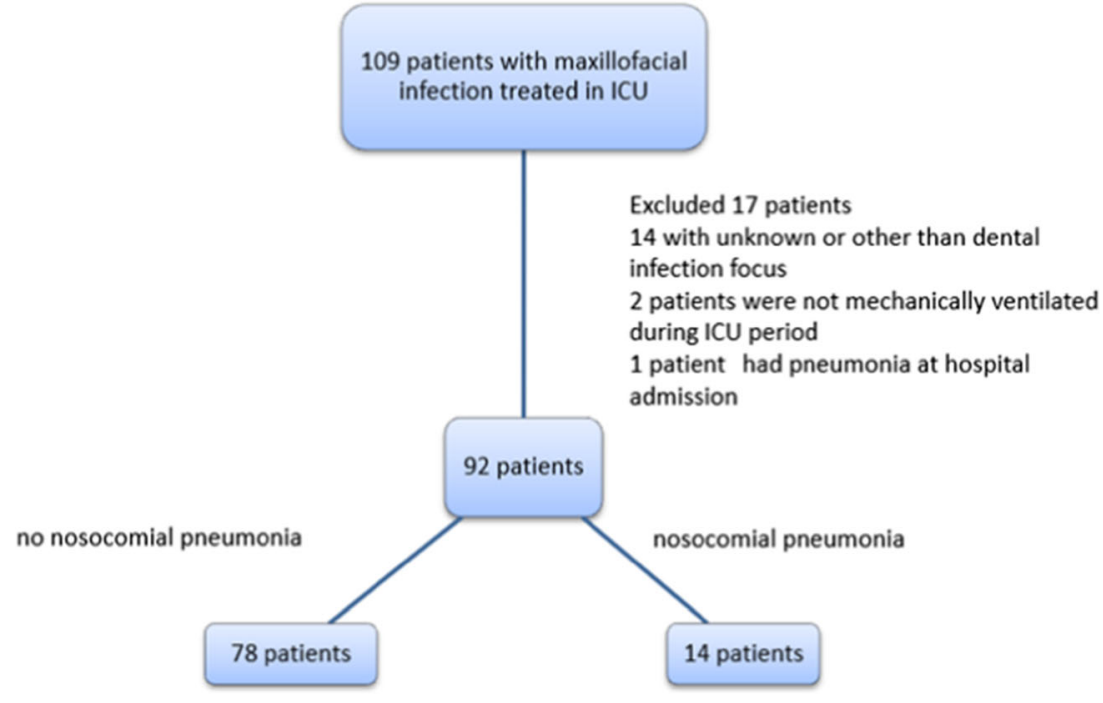

Table 1 Descriptive statistics of 92 odontogenic infection patients requiring intensive care

\begin{tabular}{|c|c|c|}
\hline \multicolumn{3}{|c|}{ Age (years) } \\
\hline Range & \multicolumn{2}{|l|}{18 to 88} \\
\hline Mean & \multicolumn{2}{|l|}{45} \\
\hline \multirow[t]{2}{*}{ Median } & \multicolumn{2}{|l|}{43} \\
\hline & $n$ & $\%$ of 92 patients \\
\hline \multicolumn{3}{|l|}{ Gender } \\
\hline Male & 63 & 68 \\
\hline Female & 29 & 32 \\
\hline \multicolumn{3}{|l|}{ Smoking } \\
\hline Yes & 30 & 33 \\
\hline No & 62 & 67 \\
\hline \multicolumn{3}{|c|}{ Heavy alcohol and/or drug user } \\
\hline Yes & 11 & 12 \\
\hline No & 81 & 88 \\
\hline \multicolumn{3}{|l|}{ Diabetes } \\
\hline Yes & 9 & 10 \\
\hline No & 83 & 90 \\
\hline \multicolumn{3}{|c|}{ Chronic pulmonary disease } \\
\hline Yes & 12 & 13 \\
\hline No & 80 & 87 \\
\hline \multicolumn{3}{|c|}{ Re-intubation } \\
\hline Yes & 6 & 7 \\
\hline No & 86 & 93 \\
\hline \multicolumn{3}{|c|}{ Duration of ventilation treatment (days) } \\
\hline Range & 0 to 31 & \\
\hline Mean & 4 & \\
\hline Median & 3 & \\
\hline \multicolumn{3}{|c|}{ Length of hospital stay (days) } \\
\hline Range & 2 to 36 & \\
\hline Mean & 7.3 & \\
\hline Median & 6 & \\
\hline
\end{tabular}

addition to DM patients, there were single patients suffering from pancreatic cancer, dilated cardiomyopathy, liver cirrhosis, and colitis ulcerosa.

Pneumonia was clinically detected and radiologically confirmed in 14 of 92 patients (15\%). It occurred between postoperative day 2 and 6 (mean 3 days, median 3 days). Four patients with pneumonia (29\%) were diagnosed between 24 and $48 \mathrm{~h}$ of treatment at the ICU, and two of the patients had been extubated at the time of diagnosis. Diagnosis was confirmed with a chest x-ray in 13 patients and with computer tomography (CT) in one patient. Pneumonia diagnosis led to antibiotic medication change in 10 of 14 patients (71\%). In all cases, antibiotic changes were made between beta-lactams; in one case clindamycin and in another case fluconazole was added to beta-lactams.

Associations between explanatory, predictor and additional variables and pneumonia are presented in Table 2 Pneumonia was more common in re-intubated patients $(p=$ 0.004). Three out of four of these patients were diagnosed prior to re-intubation. Duration of mechanical ventilation $(p=0.028)$ and LOHS $(p=0.002)$ were significantly longer in patients with pneumonia. Pneumonia was significantly more common in smokers than in non-smokers $(30 \%$ vs. $8 \%, p=0.011)$, and the majority of patients with pneumonia were smokers ( 9 of 14, 64\%).

In multivariate analysis with binomial logistic regression, our model was statistically significant, $\chi^{2}=14.469, p=0.043$, and showed an excellent level of discrimination, with a value of 0.804 for the area under the receiver-operating characteristics curve and 0.254 for the Nagelkerke $R^{2}$. Of the six predictor variables, only two were statistically significant: smoking and re-intubation, as shown in Table 3.

Eight patients received late tracheostomy during later ICU care; five of these patients had pneumonia $(p=0.002)$. In three of the five patients, pneumonia diagnosis was made prior to tracheostomy; however, none of the patients received 
Table 2 Associations between explanatory, predictor, and additional variables and pneumonia in 92 odontogenic infection patients

\begin{tabular}{|c|c|c|c|c|c|c|c|}
\hline & \multicolumn{3}{|c|}{ Patients with pneumonia } & \multicolumn{3}{|c|}{ Patients without pneumonia } & \multirow[b]{2}{*}{$p$ value } \\
\hline & $n$ & $\%$ & & $n$ & $\%$ & & \\
\hline All & 14 & 15 & & 78 & 85 & & \\
\hline \multicolumn{8}{|l|}{ Age (years) } \\
\hline Range & 21 to 79 & & & 18 to 88 & & & \\
\hline Mean & 48 & & & 44 & & & \\
\hline \multirow[t]{2}{*}{ Median } & 46 & & & 40 & & & 0.363 \\
\hline & $n$ & $\%$ & $\%$ of patients with pneumonia & $n$ & $\%$ & $\%$ of patients without pneumonia & \\
\hline \multicolumn{8}{|l|}{ Gender } \\
\hline Male & 9 & 14 & 64 & 54 & 86 & 69 & \\
\hline Female & 5 & 17 & 36 & 24 & 83 & 31 & 0.759 \\
\hline \multicolumn{8}{|l|}{ Smoking } \\
\hline Yes & 9 & 30 & 64 & 21 & 70 & 27 & \\
\hline No & 5 & 8 & 36 & 57 & 92 & 73 & 0.011 \\
\hline \multicolumn{8}{|c|}{ Heavy alcohol and/or drug user } \\
\hline Yes & 1 & 9 & 7 & 10 & 91 & 13 & \\
\hline No & 13 & 16 & 93 & 68 & 84 & 87 & 1.000 \\
\hline Diabetes & 2 & 22 & 14 & 7 & 78 & 9 & 0.622 \\
\hline Asthma/COPD* & 3 & 25 & 21 & 9 & 75 & 12 & 0.384 \\
\hline \multicolumn{8}{|l|}{ Re-intubation } \\
\hline Yes & 4 & 67 & 29 & 2 & 33 & 3 & \\
\hline No & 10 & 12 & 71 & 76 & 88 & 97 & 0.004 \\
\hline \multicolumn{8}{|c|}{ Duration of ventilation treatment (days) } \\
\hline Range & 1 to 31 & & & 0 to 14 & & & \\
\hline Mean & 8.5 & & & 3.1 & & & \\
\hline Median & 6.5 & & & 2 & & & 0.028 \\
\hline \multicolumn{8}{|c|}{ Length of hospital stay (days) } \\
\hline Range & 4 to 31 & & & 2 to 36 & & & \\
\hline Mean & 11.6 & & & 6.6 & & & \\
\hline Median & 10 & & & 5.5 & & & 0.002 \\
\hline
\end{tabular}

*COPD chronic obstructive pulmonary disease

tracheostomy principally because of pneumonia. All tracheostomies were performed due to prolonged ventilation by intubation and/or intensive swelling that was subsiding slower than anticipated.

Tracheal micro-organism aspirates were taken from 10 (71\%) of 14 pneumonia patients, and in 9 cases, the microorganism was identified in respiratory culture (Table 4). The same bacteria were detected in OI and tracheal aspirate only in one patient. The detected bacteria had no significant resistance to antibiotics, though it is not uncommon that bacteria found in aspirates of nosocomial pneumonia patients are resistant to common antibiotics.

Overall mortality was $2 \%$ (2 of 92) during hospital care. Both deceased patients suffered from secondary pneumonia. Thus, 14\% (2 of 14) of patients with pneumonia died during the ICU period following OI.

\section{Discussion}

The purpose of this study was to clarify the occurrence and characteristics of pneumonia as a nosocomial infection in patients treated for deep OI. Our hypothesis was that pneumonia occurrence is rather high in OI patients in general and that treatment-related factors can be detected. The hypothesis was confirmed partially. Pneumonia occurred in no more than $15 \%$ of patients treated for deep OIs under general anaesthesia and requiring intensive care. Duration of mechanical ventilation $(p=0.028)$ and LOHS $(p=0.002)$ were significantly related to pneumonia, which are in concordance with previous observations. As a notable finding, smokers had a significant risk for nosocomial pneumonia $(p=0.011)$.

Studies focusing on OI patients' nosocomial pneumonias are scarce, and occurrence of pneumonia has been shown to be 
Table 3 Results of the binomial logistic regression model with occurrence of pneumonia as the dependent variable

\begin{tabular}{lllll}
\hline Predictor variable & $B$ & $p$ value & OR & $95 \%$ confidence interval \\
\hline Gender, male & -0.779 & 0.302 & 0.459 & $0.105-2.013$ \\
Age & & & & \\
$\quad$ Lowest tertile (ref) & & & & \\
Middle tertile & 0.310 & 0.720 & 1.364 & $0.250-7.430$ \\
Highest tertile & 0.262 & 0.754 & 1.300 & $0.252-6.705$ \\
$\quad$ Smoking & 1.469 & 0.049 & 4.344 & $1.007-18.730$ \\
Re-intubation & 2.196 & 0.038 & 8.990 & $1.135-71.178$ \\
Diabetes mellitus & 0.871 & 0.415 & 2.390 & $0.294-19.431$ \\
Pulmonary disease & 0.219 & 0.809 & 1.245 & $0.211-7.336$ \\
\hline
\end{tabular}

moderate. In the study of Sittitrai et al. [2], pneumonia occurred in 5\% of patients with deep neck infections; however, the study focused on immunosuppressive patients and included infections with other than odontogenic aetiologies as well. The study of Ylijoki et al. [14] reported a 1\% rate of pneumonia in OI patients, but the number of patients requiring ICU treatment was low. In the recent study of Velhonoja et al. [9], $6 \%$ of patients with deep neck infection had pneumonia as an infection complication. The occurrence in the present study is higher $(15 \%)$, which is explained by the inclusion criteria. We only included patients requiring postoperative mechanical ventilation. As mentioned earlier, ICU patients' hospitalassociated pneumonia prevalence is $5-40 \%$ in the general patient population with diverse reasons for intensive care [16]. Considering the fact that all patients in the present study had increased risk for pneumonia due to difficult airway management and deep oral and neck infections leading to ICU stay, it is surprising that pneumonia occurrence was not higher than presented. In addition, it is noteworthy that the same bacteria were detected in tracheal aspirate and odontogenic pus in only one out of nine patients for whom a sample was available
(Table 4). All patients who died during hospital stay had pneumonia prior to death. Ventilation-associated pneumonia (VAP) was the most common infection and cause of death in trauma patients [21], and in a similar population, VAP mortality was reported to be $12 \%$ [17]. However, pneumonia in the present study is rather nosocomial than VAP; our results are in concordance with these previous results. The estimated mortality of VAP is around $10 \%$, but with surgical ICU patients, it is higher [16]. All-cause mortality associated with VAP is reported to be as high as $50 \%$, although the rate is questioned and previous studies from the nineties have reported conflicting results [16]. Therefore, the risk of pneumonia should be minimized.

Prolonged mechanical ventilation raises the risk for pneumonia [22, 23], the risk peaking between days 5 and 9 [16], and pneumonia patients have a distinctly longer ICU stay [16] and also LOHS [23]. Up to $63 \%$ of pneumonias develop within $48 \mathrm{~h}$ of mechanical ventilation [23]. Our results are in line with the earlier findings; duration of mechanical ventilation was markedly longer among pneumonic patients and was related to pneumonia $(p=0.028)$. In all patients, the reason for mechanical ventilation postoperatively was narrowed laryngeal space, and it was estimated in collaboration between oral and maxillofacial surgeons and anaesthesiologists. Previously, it has also been shown that re-intubation episodes predispose to pneumonia $[19,20,22]$. Although re-intubation rates were low in our study, it should be noted that $4(67 \%)$ out of 6 re-intubated patients had pneumonia, and $29 \%$ of all pneumonia patients were re-intubated during ICU stay. However, it must be emphasized that in three out of four patients with pneumonia, it was diagnosed before re-intubation, and the remaining patient received a pneumonia diagnosis on the same day that re-intubation was performed. All six re-intubations were performed because of breathing difficulties caused by narrowed laryngeal space due to persistent infection or respiratory failure. These re-intubated patients had a
Table 4 Comparison of odontogenic infection microorganisms and tracheal aspirates in 9 patients

\begin{tabular}{ll}
\hline Micro-organism in odontogenic infection pus & Micro-organism in tracheal aspirate \\
\hline Streptococcus anginosus, Prevotella intermedia & Serratia marcescens, Candida dubliniensis \\
Anaerobic gram + cocci & Candida albicans \\
Anaerobic gram + cocci & Respiratory tract normal flora \\
Streptococcus anginosus, Prevotella intermedia, anaerobic & Moraxella catarrhalis \\
gram - rod & \\
Streptococcus anginosus, Candida albicans, anaerobic mixed & Respiratory tract normal flora, Candida \\
flora, Actinomyces turicensis & albicans \\
Not taken & Coagulase-negative staphylococcus \\
Anaerobic gram - rods, oral normal flora & Klebsiella oxytoca, Klebsiella pneumoniae \\
Streptococcus anginosus, anaerobic mixed flora & (gram - anaerobic rods) \\
Streptococcus anginosus, anaerobic mixed flora & Streptococcus anginosus, Staphylococcus \\
\hline
\end{tabular}


severe, deep OI and a narrowed airway that often lead to pneumonia which developed before reintubation. In addition, pneumonia in these patients may also have contributed to the need for reintubation. Thus, pneumonia was not the result of re-intubation, but rather it occurred in patients with a constricted airway. Declining of infection, subsiding of swelling, and an open airway should be ensured prior to extubation to avoid the need for reinstitution of ventilatory support, especially in a population such as the present one in which intubation is often challenging. On the other hand, a longer mechanical ventilation period raises the risk of pneumonia, so effective early infection treatment and timing of extubation are crucial. Smooth communication between the ICU physician and the oral and maxillofacial surgeon is essential when making treatment decisions about extubation or continuation of mechanical ventilation.

Only one of the 92 patients had tracheostomy performed at the primary stage of OI treatment; this patient did not develop nosocomial pneumonia during the ICU period. Tracheostomy has been thought to decrease complications related to longer intubation times in patients with deep neck infection according to the study of Tapiovaara et al. [24]. In that study, patients were treated mostly for otolaryngological infections. In lower neck infections, tracheostomy may be considered, and patients can be transferred to the ward earlier. With regard to pneumonia, a randomized and controlled study in mechanically ventilated adult ICU patients found no difference in the incidence of VAP between early and late tracheostomy patients [25]. According to our results, routine tracheostomy in all OI patients requiring ICU treatment to prevent secondary pneumonia is unnecessary and would expose patients pointlessly to the disadvantages of tracheostomy. In smokers it may be advisable to consider early tracheostomy if patient's clinical status is poor and duration of mechanical ventilation is expected to continue long.

In our study, pneumonia developed in $30 \%$ of smokers, while only $8 \%$ of non-smokers had pneumonia. In addition, almost two-thirds (64\%) of pneumonia patients were smokers. In a recent publication by Du et al. [26], smoking was associated with a longer ICU period in spine trauma patients, but it was not found to increase the risk for pneumonia [26]. The authors stated that the small sample size may have caused a false-negative bias [26]. Smoking has also been found to correlate with the LOHS in patients with deep neck infections, thereby increasing the risk for complications [27]. Therefore, preventing the risk of pneumonia, especially in smokers, is warranted.

DM or previous chronic pulmonary disease did not predispose to pneumonia in the present study. Although studies of OI and pneumonia remain scarce, in different OI settings both $\mathrm{DM}$ and COPD have been identified as significant risk factors for nosocomial pulmonary infections, and patients with DM have been shown to have different and rarer bacterial strains and a significantly higher drug resistance rate than patients without DM [28-31]. In large cohorts, the pneumonia risk in DM patients seems obvious; López-de-Andrés et al. [32] reported a $21 \%$ higher incidence of postoperative pneumonia in DM patients than in non-diabetic patients in a cohort of over 100,000 patients, although contradictory results have also been reported [33, 34]. Associations between chronic pulmonary disease or DM and pneumonia were not verified in our study; however, the number of patients with a history of these diseases was too low for reliable statistical evaluation.

Interestingly, none of the pneumonia patients were iatrogenically immunosuppressed. One pneumonia patient had labile DM type 1, which predisposes to infections in general, one patient had DM type 2, and one patient had dilated cardiomyopathy. The present findings that immunosuppressive patients were not exposed to pneumonia and that patients had low susceptibility to infections in general may be due to effective treatment in early infections. It is a common practice that all dental infections must be treated before any immunosuppressive medication (i.e., biological medications or cytostatic treatment) is started. In addition, patients are informed that dental hygiene and treatment are important during use of these medications.

Prevention of nosocomial pneumonia in mechanically ventilated patients is crucial. The best way to prevent VAP is to critically assess the need for mechanical ventilation and evaluate daily the conditions for extubation. According to our hospital protocol, it is recommended to use as light sedation as possible, the head of the bed should be elevated to prevent micro-aspiration of stomach contents, and the collection of secretion above the cuff must be minimized and the cuff pressure checked and optimized to $20-30 \mathrm{~cm} \mathrm{H}_{2} \mathrm{O}$. Careful oral care combined with use of mouth rinse, such as hydrogen peroxide [35], is a local and easy treatment method and is particularly well-suited for OIs to prevent colonization of oral pathogens on the endotracheal tube. The use of oral chlorhexidine (CHX) is controversial and unclear in preventing pneumonia. The study of Papazian et al. [16] noted that the use of oral CHX may not be recommended because no association has been shown between CHX use in oral care and lower rates of pneumonia; on the contrary, it seems to increase mortality rates perhaps due to aspiration. Then again, a systematic review by Hua [36] has shown that the use of oral CHX decreases the risk of VAP from 25 to $19 \%$.

In the present study, clinical suspicion of pneumonia was verified by chest $\mathrm{x}$-ray in 13 patients and by $\mathrm{CT}$ in one patient. The determination of pneumonia in patients treated for another severe infection in ICU is challenging, and diagnosis based solely on imaging is not reliable [37]. ICU patients' radiographs are most often performed in a lying position, rendering interpretation of the image more difficult. It is known that chest $\mathrm{x}$-ray is not sensitive or specific in VAP diagnostics; in the study of Papazian et al. [16], two patients' chest x-rays 
were falsely negative. More precise criteria than clinical suspicion and radiological confirmation of pneumonia were not used; therefore, misdiagnosis or incorrect diagnosis of pneumonia may have occurred. Thus, this can be considered a clear weakness of the study. In addition, data of possible comorbidity of the pneumonia patients, assessment of lung function capacity, as well as details of mechanical ventilation would have clarified the risks of VAP more. In deep OIs, the intubation procedure is usually challenging due to swelling, and there is also a possibility of aspiration of, e.g. bursting pus. We were unable to analyse these factors due to the retrospective nature of the study. To clarify more the risk of pneumonia in OI patients, more extensive patient data including tracheal aspirates, evaluation of intubation circumstances, and more detailed data of ICU period would be required.

\section{Conclusion}

Overall, $15 \%$ of all OI patients treated in ICU with mechanical ventilation had nosocomial pneumonia. The study shows that challenging airway circumstances, prolonged restricted airway, lengthy mechanical ventilation, and smoking predispose to nosocomial pneumonia in the OI population. The risk of pneumonia can be reduced by early and prompt treatment of OI, thereby decreasing the duration of mechanical ventilation.

Funding information Open access funding provided by University of Helsinki including Helsinki University Central Hospital. The study was not supported by any funding source. Tuukka Puolakka received a research grant from Vyborg's institution of tuberculosis and Johanna Snäll grants from the Paulo Foundation and the Helsinki University Hospital Fund.

\section{Compliance with ethical standards}

Conflict of interests The authors declare that they have no conflicts of interest.

Ethical approval The study was approved by the Internal Review Board of the Head and Neck Center, Helsinki University Hospital, Helsinki, Finland (58/2020).

Open Access This article is licensed under a Creative Commons Attribution 4.0 International License, which permits use, sharing, adaptation, distribution and reproduction in any medium or format, as long as you give appropriate credit to the original author(s) and the source, provide a link to the Creative Commons licence, and indicate if changes were made. The images or other third party material in this article are included in the article's Creative Commons licence, unless indicated otherwise in a credit line to the material. If material is not included in the article's Creative Commons licence and your intended use is not permitted by statutory regulation or exceeds the permitted use, you will need to obtain permission directly from the copyright holder. To view a copy of this licence, visit http://creativecommons.org/licenses/by/4.0/.

\section{References}

1. Qu L, Liang X, Jiang B, Qian W, Zhang W, Cai X (2018) Risk factors affecting the prognosis of descending necrotizing Mediastinitis from Odontogenic infection. J Oral Maxillofac Surg 76(6):1207-1215

2. Sittitrai P, Srivanitchapoom C, Reunmakkaew D (2018) Deep neck infection in patients with and without human immunodeficiency virus: a comparison of clinical features, complications, and outcomes. Br J Oral Maxillofac Surg 56(10):962-967

3. Huang TT, Liu TC, Chen PR, Tseng FY, Yeh TH, Chen YS (2004) Deep neck infection: analysis of 185 cases. Head Neck 26(10):854 860. https://doi.org/10.1002/hed.20014

4. Chi TH, Tsao YH, Yuan CH (2014) Influences of patient age on deep neck infection: clinical etiology and treatment outcome. Otolaryngol Head Neck Surg 151(4):586-590

5. Seppänen L, Lauhio A, Lindqvist $C$, Suuronen R, Rautemaa R (2008) Analysis of systemic and local odontogenic infection complications requiring hospital care. J Infect 57(2):116-122

6. Seppänen L, Rautemaa R, Lindqvist C, Lauhio A (2010) Changing clinical features of odontogenic maxillofacial infections. Clin Oral Investig 14(4):459-465

7. Glen P, Morrison J (2016) Diffuse descending necrotising mediastinitis and pleural empyema secondary to acute odontogenic infection resulting in severe dysphagia. BMJ Case Rep 2016: bcr2015212145. Published 2016 Mar 24. https://doi.org/10.1136/ bcr-2015-212145

8. Haraden BM, Zwemer FL (1997) Descending necrotizing mediastinitis: complication of a simple dental infection. Ann Emerg Med 29(5):683-686

9. Velhonoja J, Lääveri M, Soukka T, Irjala H, Kinnunen I (2020) Deep neck space infections: an upward trend and changing characteristics. Eur Arch Otorhinolaryngol 277(3):863-872

10. Cariati P, Cabello-Serrano A, Monsalve-Iglesias F, Roman-Ramos M, Garcia-Medina B (2016) Meningitis and subdural empyema as complication of pterygomandibular space abscess upon tooth extraction. J Clin Exp Dent 8(4):e469-e472

11. Greenstein A, Witherspoon R, Leinkram D, Malandreni M (2015) An unusual case of a brain abscess arising from an odontogenic infection. Aust Dent J 60(4):532-535

12. Maria A, Rajnikanth K (2010) Cervical necrotizing fasciitis caused by dental infection: a review and case report. Natl J Maxillofac Surg 1(2):135-138

13. Noy D, Rachmiel A, Dan LF, Emodi O (2015) Lemierre's syndrome from odontogenic infection: review of the literature and case description. Ann Maxillofac Surg 5(2):219-225

14. Ylijoki S, Suuronen R, Jousimies-Somer H, Meurman JH, Lindqvist C (2001) Differences between patients with or without the need for intensive care due to severe odontogenic infections. J Oral Maxillofac Surg 59(8):867-872

15. Vincent JL, Rello J, Marshall J, Silva E, Anzueto A, Martin CD, Moreno R, Lipman J, Gomersall C, Sakr Y, Reinhart K, EPIC II Group of Investigators (2009) International study of the prevalence and outcomes of infection in intensive care units. Jama 302(21): 2323-2329

16. Papazian L, Klompas M, Luyt CE (2020) Ventilator-associated pneumonia in adults: a narrative review. Intensive Care Med 46(5):888-906. https://doi.org/10.1007/s00134-020-05980-0

17. Michetti CP, Fakhry SM, Ferguson PL, Cook A, Moore FO, Gross R, AAST Ventilator-Associated Pneumonia Investigators (2012) Ventilator-associated pneumonia rates at major trauma centers compared with a national benchmark: a multi-institutional study of the AAST. J Trauma Acute Care Surg 72(5):1165-1173 
18. Decelle L, Thys F, Zech F, Verschuren F (2013) Ventilationassociated pneumonia after intubation in the prehospital or the emergency unit. Eur J Emerg Med 20(1):61-63

19. Torres A, Gatell JM, Aznar E, el-Ebiary M, Puig de la Bellacasa J, González J, Ferrer M, Rodriguez-Roisin R (1995) Re-intubation increases the risk of nosocomial pneumonia in patients needing mechanical ventilation. Am J Respir Crit Care Med 152(1):137141

20. Gao F, Yang LH, He HR, Ma XC, Lu J, Zhai YJ, Guo LT, Wang X, Zheng J (2016) The effect of reintubation on ventilator-associated pneumonia and mortality among mechanically ventilated patients with intubation: a systematic review and meta-analysis. Heart Lung 45(4):363-371

21. Kung SC, Lin WT, Tsai TC, Lin MH, Chang CH, Lai CC, Chao CM (2017) Epidemiologic characteristics and outcomes of major trauma patients requiring prolonged mechanical ventilation. Medicine (Baltimore) 96(52):e9487

22. Wolkewitz M, Palomar-Martinez M, Alvarez-Lerma F, OlaecheaAstigarraga P, Schumacher M (2019) Analyzing the impact of duration of ventilation, hospitalization, and ventilation episodes on the risk of pneumonia. Infect Control Hosp Epidemiol 40(3):301-306

23. Rello J, Ollendorf DA, Oster G, Vera-Llonch M, Bellm L, Redman R, Kollef MH, VAP Outcomes Scientific Advisory Group (2002) Epidemiology and outcomes of ventilator-associated pneumonia in a large US database. Chest 122(6):2115-2121

24. Tapiovaara L, Bäck L, Aro K (2017) Comparison of intubation and tracheotomy in patients with deep neck infection. Eur Arch Otorhinolaryngol 274(10):3767-3772

25. Terragni PP, Antonelli M, Fumagalli R, Faggiano C, Berardino M, Pallavicini FB, Miletto A, Mangione S, Sinardi AU, Pastorelli M, Vivaldi N, Pasetto A, Della Rocca G, Urbino R, Filippini C, Pagano E, Evangelista A, Ciccone G, Mascia L, Ranieri VM (2010) Early vs late tracheotomy for prevention of pneumonia in mechanically ventilated adult ICU patients: a randomized controlled trial. Jama 303(15):1483-1489

26. Du JY, Weinberg DS, Moore TA, Vallier HA (2020) Smoking is associated with longer intensive care unit stays in spine trauma patients. J Orthop Trauma 34(7):e250-e255. https://doi.org/10. 1097/BOT.0000000000001739

27. Barber BR, Dziegielewski PT, Biron VL, Ma A, Seikaly H (2014) Factors associated with severe deep neck space infections: targeting multiple fronts. J Otolaryngol Head Neck Surg 43(1):35
28. Xu Y, Lai C, Xu G, Meng W, Zhang J, Hou H, Pi H (2019) Risk factors of ventilator-associated pneumonia in elderly patients receiving mechanical ventilation. Clin Interv Aging 14:1027-1038

29. Chang L, Dong Y, Zhou P (2017) Investigation on risk factors of ventilator-associated pneumonia in acute cerebral hemorrhage patients in intensive care unit. Can Respir J 2017:7272080-7272084. https://doi.org/10.1155/2017/7272080

30. Wu HP, Chu CM, Lin CY et al (2016) Liver cirrhosis and diabetes mellitus are risk factors for Staphylococcus aureus infection in patients with healthcare-associated or hospital-acquired pneumonia. Pulm Med 2016:4706150-4706156. https://doi.org/10.1155/2016/ 4706150

31. Rubin BK et al (2011) Respiratory care year in review 2010: Part 1. Asthma, COPD, pulmonary function testing, ventilator-associated pneumonia. Respir Care 56(4):488

32. López-de-Andrés A, Perez-Farinos N, de Miguel-Díez J et al (2019) Type 2 diabetes and postoperative pneumonia: an observational, population-based study using the Spanish Hospital Discharge Database, 2001-2015. PLoS One 14(2):e0211230 Published 2019 Feb 6. https://doi.org/10.1371/journal.pone.0211230

33. de Oliveira TFL et al (2011) Factors associated with nosocomial pneumonia in hospitalized individuals. Rev Assoc Med Bras 57(6): 630-636

34. NanZhu Y, Xin L, Xianghua Y, Jun C, Min L (2019) Risk factors analysis of nosocomial pneumonia in elderly patients with acute cerebral infraction. Medicine (Baltimore) 98(13):e15045. https:// doi.org/10.1097/MD.0000000000015045

35. Nobahar M, Razavi MR, Malek F, Ghorbani R (2016) Effects of hydrogen peroxide mouthwash on preventing ventilator-associated pneumonia in patients admitted to the intensive care unit. Braz $\mathrm{J}$ Infect Dis 20(5):444-450

36. Hua F, Xie H, Worthington HV, Furness S, Zhang Q, Li C (2016) Oral hygiene care for critically ill patients to prevent ventilatorassociated pneumonia. Cochrane Database Syst Rev 10(10): CD008367. Published 2016 Oct 25. https://doi.org/10.1002/ 14651858.CD008367.pub3

37. Lefcoe MS, Fox GA, Leasa DJ, Sparrow RK, McCormack DG (1994) Accuracy of portable chest radiography in the critical care setting - diagnosis of pneumonia based on quantitative cultures obtained from protected brush catheter. Chest 105(3):885-887

Publisher's note Springer Nature remains neutral with regard to jurisdictional claims in published maps and institutional affiliations. 